\title{
Pattern and characteristics of advanced cancer patients admitted to hospices in Italy
}

\author{
Sebastiano Mercadante - Alessandro Valle • \\ Silvana Sabba • Antonio Orlando • Francesca Guolo • \\ Loretta Gulmini • Sara Ori • Rosanna Bellingardo • \\ Alessandra Casuccio
}

Received: 19 March 2012 / Accepted: 18 September 2012 /Published online: 4 October 2012

(C) Springer-Verlag Berlin Heidelberg 2012

\begin{abstract}
The aim of this multicenter study was to assess the pattern and the characteristics of advanced cancer patients admitted to hospices. A consecutive sample of patients admitted in a period of 6 months was taken into consideration. Two hundred thirty-six patients admitted to hospices were consecutively assessed. Ninety-six percent of patients were admitted in acute hospital in the previous 3 months, with a mean time spent in hospital of 34.5 days, and $47 \%$ of patients had received chemotherapy the month before hospice admission. Thirty-four percent of patients for
\end{abstract}

S. Mercadante $(\bowtie) \cdot R$. Bellingardo

Pain Relief and Palliative Care Unit, La Maddalena Cancer Center, Palermo, Italy

e-mail: terapiadeldolore@lamaddalenanet.it

S. Mercadante $\cdot$ R. Bellingardo

Department of Palliative Medicine, University of Palermo,

Palermo, Italy

S. Sabba

Hospice ASL TO4,

Sede legale: via Po $n$,

11-10034 Chivasso, Turin, Italy

L. Gulmini $\cdot$ S. Ori

Hospice "Le onde e il mare",

Codigoro, Ferrara, Italy

\section{A. Valle}

Hospice FARO,

Strada Comunale S. Vito Revigliasco, 34,

Turin, Italy

\section{A. Orlando $\cdot$ F. Guolo}

Hospice Casa dei Gelsi,

Treviso, Italy

\section{A. Casuccio}

Department of Clinical Neuroscience, University of Palermo, Palermo, Italy whom data were available had significant persistent pain, and $44 \%$ of them presented episodes of breakthrough pain. Sixty-one percent of patients were receiving opioid drugs at admission, and $70 \%$ the day before death, with parenteral morphine and transdermal fentanyl being the opioids most frequently administered. The mean admission time in hospice was 18.4 days. Eighty-six percent died in hospice. Palliative sedation was performed in $25 \%$ of patients who died in hospice. The short survival and the number of patients dying in hospice were the principal finding, as it appears that hospice admission is only one way for end of life treatments. Patients receive specialized palliative care only for 2-3 weeks before death, implying an inacceptable timing for patients with several problems presumed to be present early during the course of disease. Data from hospice activities in Italy strongly suggest to spread palliative care in other settings, other than home care and hospice, to intercept oncologic patients in their disease trajectory early.

Keywords Palliative care $\cdot$ Hospice $\cdot$ Advanced cancer

\section{Introduction}

Palliative care was initiated in Italy at the end of the 1970s as a natural progression from an interest in pain control to a growing importance to resolve other problems for cancer patients, particularly in the advanced stage of disease. During 1980 s, various organizations, usually charities or noprofit organizations, developed different models of home care for patients with advanced stage disease [1]. In the last decade, there have been investments in the field of community care and health districts under the input of new legislations, and new resources from the health care system have been allocated for palliative care. Active centers involved in 
palliative care progressively increased with a large distribution in Northern Italy, and more than 200 hospices are now available for cancer and non cancer palliative care population [2]. However, data regarding the clinical activity in traditional hospices have never been assessed. The only existing data regard an inpatient acute palliative care unit, which represent an exception, because it provides supportive care during all the entire period of disease, only a minority of patients are admitted in the final phase of life, and most patients are discharged home or will continue their oncologic treatments [3, 4]. Italy represents a unique country for its specificity in cultural issues, attitudes, organization, and health care system. In fact, palliative care has been recognized by a specific law only in the last years, and negative attitudes for opioid consumption are well known. Moreover, the sociocultural tissue and health care system cannot be considered equal to other European countries.

The aim of this multicenter study was to assess the pattern and the characteristics of advanced cancer patients admitted to traditional hospices with long-term facilities, as well as their treatment at the end of life.

\section{Methods}

Four hospices participated into the study. These four hospices were selected according to a similar number of beds, admissions, structures, territorial aspects, users, and health care professionals, and could be considered representative of Italian hospices, which are prevalently distributed in the North. A consecutive sample of patients admitted in a period of 6 months (from January to June 2011) was taken into consideration. Data of hospice admissions were prospectively collected, and a specific form was designed for this purpose. Informed consent from patients or relatives and ethical committee approval were obtained. Epidemiological data, including marital status and caregiver availability, were recorded. The number of admissions and days spent in hospital in the last 3 months, as well the reasons for hospital admission and days elapsed from the last chemotherapy were collected.

Patients were divided according to the principal indication for hospice admission, including social problems, uncontrolled pain, uncontrolled symptoms other than pain, end of life care, and others. Once patients were admitted, they were asked about pain and its intensity (on a numerical scale of 0-10), breakthrough pain and its intensity, and analgesics prescribed. These data were also collected on the day before death. The duration of hospice admission, the frequency of discharge home, or frequency of death in hospice were recorded. Finally, the use of palliative sedation, its duration, and drugs used on the day of death were recorded.
Statistical analysis

Statistical analysis of quantitative and qualitative data, including descriptive statistics, was performed for all the items. Continuous data were expressed as mean \pm standard deviation, unless otherwise specified. The linear regression and multiple logistic regression models were used to analyze the relation between the mean admission time in hospice and continuous and categorical variables, respectively. Data were analyzed by the Epi Info software (version 6.0, CDC, Atlanta, GA, US) and the SPSS Software 14.0 version (SPSS, Inc., Chicago, Ill, US). All $P$ values were two-sided and $P$ values less than 0.05 were considered to indicate statistical significance.

\section{Results}

The characteristics of patients are described in Table 1. The civil status was available in 223 patients: 113 patients were married, 70 patients were widow, 24 patients were single, 14 patients were divorced, and 2 were common-law partners. Caregivers were available for 215 patients and were spouse 65 , adult children 102 , other relatives 32 , friends or healthcare workers 14, and nonrelated 2. Of the 211 patients who provided an answer, 203 patients $(96 \%)$ had been admitted in acute hospital in the previous 3 months, with a mean time spent in hospital of 34.5 (SD 37.8) days: 110 patients were admitted once, 73 patients twice, 18 patients three times, and 2 patients four times. One hundred twelve patients (47\%) had received chemotherapy the month before hospice admission.

Indications for hospice admission, available in 205 patients, are presented in Table 2. Seventy-eight of 230 (34\%) patients for whom data were available had significant persistent pain ( $>4 / 10$ on a numerical scale), and $44 \%$ of them presented episodes of breakthrough pain. The mean
Table 1 Characteristics of advanced cancer patients admitted to hospice

\begin{tabular}{ll}
\hline $\begin{array}{l}\text { Number of } \\
\text { patients } \\
\begin{array}{l}\text { Gender } \\
\text { (male/female) }\end{array} \\
\text { Age }\end{array}$ & 236 \\
& 73 (range 37-100, \\
SD 11.6) \\
Primary diagnosis & \\
Gastrointestinal & 47 \\
Genitourinary & 46 \\
Lung & 44 \\
Liver & 22 \\
Breast & 18 \\
Head-neck & 15 \\
Pancreas & 13 \\
Others & 27 \\
\hline
\end{tabular}


Table 2 Principal indications for hospice admission (available in 205 patients)

\begin{tabular}{lr}
\hline Control of other symptoms & 103 \\
Pain control & 49 \\
Cachexia & 20 \\
Anemia & 14 \\
Unspecified surgical problems & 6 \\
Social problems & 5 \\
Reassessment (laboratory or & 6 \\
imaging) & 1 \\
Opioid toxicity & 1 \\
Chemotherapy & \\
\hline
\end{tabular}

pain intensity of patients who had persistent pain at hospice admission was 4.2 (SD 2.4), and the mean breakthrough pain intensity was 8 (SD 2.2). Of the entire sample of 236 patients, 144 of them $(61 \%)$ were receiving analgesic drugs. Parenteral morphine and transdermal fentanyl were the opioids most frequently administered at hospice admission (48 and 44 patients, respectively) in mean doses of $71 \mathrm{mg} /$ day, and $51.3 \mu \mathrm{g} / \mathrm{h}$, respectively. Eighteen patients were administered immediate or sustained release of oxycodone in mean doses of $60 \mathrm{mg} /$ day. Oral morphine (immediate or sustained release formulations) was administered in seven patients in mean doses of $50 \mathrm{mg} /$ day. Five patients were receiving transdermal buprenorphine in mean doses of $45 \mu \mathrm{g} / \mathrm{h}$. One patient was receiving oral hydromorphone. Nineteen patients were receiving different preparations of codeine or tramadol, and two patients were receiving non-opioid analgesics. Thirteen patients were receiving more analgesics. Ninety-six patients had a prescription of analgesics for breakthrough pain. Forty-one patients were receiving parenteral morphine (at a mean dose $14 \mathrm{mg}$, SD 14) and 27 were given oral morphine (mean a dose $14 \mathrm{mg}$, SD 10). Only two patients were receiving rapid onset opioids (transmucosal fentanyl citrate and fentanyl buccal tablet). The remaining patients were receiving tramadol, codeine, or non-opioid drugs, in different preparations.

The day before dying, 165 patients $(70 \%)$ received opioid drugs. Most of them were administered parenteral morphine (114 patients, in mean doses of $63 \mathrm{mg} /$ day) or transdermal fentanyl (28 patients, in mean doses of $48 \mathrm{mcg} / \mathrm{h}$ ). Eight patients received different formulations of oxycodone (in mean doses of $25 \mathrm{mg} /$ day), and two patients received transdermal buprenorphine. The remaining patients were receiving codeine or tramadol preparations.

The day before dying, 28 of 90 patients (31\%) with available data had episodes of breakthrough pain. The most frequent prescription was parenteral morphine while transmucosal, buccal, and sublingual fentanyl were prescribed in one, three, and two patients, respectively.
The mean admission time in hospice was 18.4 (SD 15.4) days. Two hundred two patients $(86 \%)$ died in hospice, and the remaining were discharged home.

Palliative sedation was performed for untreatable symptoms in $25 \%$ of patients who died in hospice. The main reason was a state of agitation and/or dyspnea (75\%). In 76 and $24 \%$ of cases, palliative sedations were performed giving drugs intravenously and subcutaneously, respectively. Midazolam alone or in combination was used in $87 \%$ of patients. Hyoscine was added in $9.6 \%$ of patients. Neuroleptics were administered alone or in combination in $15 \%$ of patients. Only one patient was receiving morphine without midazolam of neuroleptics. The mean duration of palliative sedation was 2.5 (SD 3.3) days.

No differences among the centers were found. In an analysis of linear and multiple logistic regression for continuous and categorical variables, no significant relationship for age, gender, marital status, caregiver, hospital admission in the last 3 months, background pain intensity, and doses of opioids was found (see Table 3). Presence of background pain and breakthrough pain episodes at hospice admission was significantly associated with short survival. Pain intensity of breakthrough pain episodes at hospice admission was significantly associated with short survival. No correlation was found with background pain intensity the day before death, opioid doses for background pain the day before death, or pain intensity of breakthrough pain episodes and before death (see Table 3).

\section{Discussion}

This study showed the pattern and the characteristics of advanced cancer patients admitted to a hospice in Italy. The first relevant findings are the short survival (about 18 days) and the number of patients dying in hospice. It is likely that these figures are even higher as some patients are often discharged home "agonic", under the pressure of relatives, according to national cultural attitudes. From these data, it appears that hospice admission is only one way for end of life treatments. Of interest, in the last 3 months before hospice admission, most of them were admitted to hospital and spent about $1 / 3$ of this period in hospital, and half of them received chemotherapy in the last month of life. Thus, home care and hospice patients receive specialized palliative care only for 2-3 weeks before death, implying an inacceptable timing for patients with several problems presumed to be present early during the course of disease. This is confirmed by the large number of hospital admissions in different acute settings expected to not providing specialized palliative care resulting in a low level of cost-effectiveness. This observation can also explain the low opioid consumption traditionally reported in Italy [5]. It is unknown whether patients were admitted to hospices from 
Table 3 Analysis of multiple regression on the relation between mean admission time in hospice and continuous and categorical variables

The dependent variable is the mean admission time in hospice. The asterisk $(*)$ indicates statistical significance $S E$ standard error

\begin{tabular}{|c|c|c|c|}
\hline Independent variable & Slope Coefficient (SE) & Coefficient of determination $R^{2}$ & $P$ \\
\hline Age & $-0.077(0.08)$ & 0.0034 & 0.382 \\
\hline Gender & $-1.678(1.74)$ & 0.0030 & 0.427 \\
\hline Marital status & $-0.798(0.54)$ & 0.9620 & 0.198 \\
\hline Caregiver & $-2.796(2.24)$ & 0.8130 & 0.216 \\
\hline $\begin{array}{l}\text { Hospital admission in the last } \\
3 \text { months }\end{array}$ & $-0.181(1.42)$ & 0.0009 & 0.665 \\
\hline $\begin{array}{l}\text { Presence of background } \\
\text { pain at hospice admission }\end{array}$ & $-7.161(2.10)$ & 0.0486 & $0.032 *$ \\
\hline $\begin{array}{l}\text { Background pain intensity } \\
\text { at hospice admission }\end{array}$ & $-0.866(0.51)$ & 0.0373 & 0.094 \\
\hline $\begin{array}{l}\text { Opioid doses for background } \\
\text { pain at hospice admission }\end{array}$ & $0.002(0.006)$ & 0.0005 & 0.786 \\
\hline $\begin{array}{l}\text { Presence of breakthrough pain } \\
\text { episodes at hospice admission }\end{array}$ & $-6.191(2.74)$ & 0.0461 & $0.041^{*}$ \\
\hline $\begin{array}{l}\text { Breakthrough pain intensity at } \\
\text { hospice admission }\end{array}$ & $-1.596(0.64)$ & 0.1157 & $0.017^{*}$ \\
\hline $\begin{array}{l}\text { Background pain intensity the } \\
\text { day before death }\end{array}$ & $-1.133(1.15)$ & 0.0459 & 0.338 \\
\hline $\begin{array}{l}\text { Opioid doses for background } \\
\text { pain the day before death }\end{array}$ & $-0.007(0.01)$ & 0.0025 & 0.528 \\
\hline $\begin{array}{l}\text { Breakthrough pain intensity } \\
\text { the day before death }\end{array}$ & $-1.769(1.15)$ & 0.1053 & 0.141 \\
\hline
\end{tabular}

inpatient settings or lack of home care availability. On the other hand, some palliative interventions, often performed in an acute setting, cannot be always provided in the hospices, contributing to late referrals. This information should be assessed in future studies to explain the pathway and timing of patients admitted to hospices.

Despite a new law has generated much interest in palliative care and pain control in Italy, also facilitating opioid prescription, institutional indications have suggested that the palliative care network should be formed by home care and hospice care only, excluding the vast majority of patients living some years or months during the course of disease. Data from this study confirm that this approach is misleading, resulting in more expenses due to inappropriate admissions in hospitals without expertise in palliative care while excluding most patients for a large period of time for prolonged periods of time, when they should need a specific help in pain and symptom management. Rather, the duration of survival of patients enrolled in hospice programs is an important measure to assess the appropriateness of timing of referral of terminally ill patients to palliative care. Patients are referred to palliative care only in the last weeks of life, as reported in this study performed in the hospice setting, and in other studies in home care setting [6-8]. This aspect was also underlined in very specialized centers in other countries [9]. In a national follow-back survey representative of Italian population, $51 \%$ of patients died at home, meaning that many patients were admitted to acute wards unfit to provide end-of-life care [10]. Finally, about $25 \%$ of patients followed at home presented severe symptom intensity for which home care was practically impossible, suggesting that the best option would probably be the admission to hospices or specialized inpatients hospital units [7]. More recently, $10 \%$ of patients followed at home were admitted to acute wards unfit to provide end-of-life care hospital [8]. On the other hand, this late referral to palliative care is also in contrast with the classical definition of palliative care provided by WHO: "Palliative care is an approach that improves the quality of life of patients and their families facing the problem associated with life-threatening illness, through the prevention and relief of suffering by means of early identification and impeccable assessment and treatment of pain and other problems, physical, psychosocial and spiritual"; "Palliative care will enhance quality of life, and may also positively influence the course of illness; is applicable early in the course of illness, in conjunction with other therapies that are intended to prolong life, such as chemotherapy or radiation therapy, and includes those investigations needed to better understand and manage distressing clinical complications" [11]. Finally, recent scientific evidence in a population subgroup confirms this WHO statement either for quality of life as well as survival. As compared with patients receiving standard care, patients receiving early palliative care had less aggressive care at the end of life but longer survival [12]. Futile treatments should be limited [13].

As expected, most patients were admitted for pain and symptom management, or indefinite cachexia, which should correspond to a terminal stage of disease. The mean age of hospice patients was relatively high. This could be explained by a specific selection of this population, possibly 
due to lack of geriatric long-term facilities in Italy. At admission, one third of patients had their pain uncontrolled, despite receiving analgesic drugs, and almost half of them had episodes of breakthrough pain. About $60 \%$ of patients were receiving opioid drugs, principally parenteral morphine and transdermal fentanyl. As expected, opioid doses were significantly less than those observed in acute palliative care units [14].

The day before dying, on average of 18 days, more patients $(70 \%)$ were receiving opioid drugs, prevalently parenteral morphine. About one third of patients presented episodes of breakthrough pain for which parenteral morphine was prescribed (not always given). The most striking data regarding the use of breakthrough medications were the poor use of rapid onset opioids, possibly due to their cost and poor confidence with the use. This confirms previous findings reported in a survey of attitudes in treating breakthrough pain in Italian hospices [15].

The frequency of palliative sedation was higher than that reported in home care patients in a previous survey (13\%) [16]. This possibly reflects a population selection bias and/ or attitudes in a more protected environment allowing timely intervention. For example, in an acute palliative care setting, the frequency of sedation was even higher, more than $50 \%$ of patients dying in the unit. This is explained by the selection of patients admitted for more distressing conditions and compelling criteria, as witnessed by the short duration of sedation [17]. Duration of palliative sedation in this study was relatively similar to that reported at home (3.5 days on average) and drug choices were similar [16].

\section{Conclusion}

Data from hospice activities in Italy strongly suggest to spread palliative care in other settings, other than home care and hospice, to intercept oncologic patients in their disease trajectory early, for example, in high volume oncologic departments, rather than restricting the action area only in the last weeks of life [2, 4, 18]. Amendments to the recent law should favor anticipation of palliative-supportive care, to correct this important gap, promoting expertise in palliative care or institution of specialized units in oncologic departments working in a multidisciplinary model of simultaneous care through all the period of disease.

Acknowledgments We are indebted with the teams of these hospices participating into this study.
Conflict of interest This research received no specific grant from any funding agency in the public, commercial, or not-for-profit sectors. Authors have full control of all primary data we agree to allow the journal to review their data if requested.

\section{References}

1. Sbanotto A, Burnhill R (1998) Palliative care in Italy: the current situation. Support Care Cancer 6:426-429

2. Zucco F (2007) Hospice in Italia, Ministero della Salute

3. Mercadante S, Villari P, Ferrera P (2003) A model of acute symptom control unit: pain relief and palliative care unit of la Maddalena cancer center. Support Care Cancer 11:114-119

4. Mercadante S, Intravaia G, Villari P, Ferrera P, David F, Casuccio A et al (2008) Clinical and financial analysis of an acute palliative care unit in an oncological department. Palliat Med 22:760-767

5. Mercadante S, Vitrano V (2010) Palliative care in Italy: problems area. Minerva Anestesiol 76:1060-1071

6. Costantini M, Toscani F, Gallucci M, Brunelli C, Miccinesi G, Tamburini $\mathrm{M}$ et al (1999) Terminal cancer patients and timing of referral to palliative care: a multicenter prospective cohort study. J Pain Symptom Manage 18:243-252

7. Peruselli C, Di Giulio P, Toscani F, Gallucci M, Brunelli C, Costantini $\mathrm{M}$ et al (1999) Home palliative care for terminal cancer patients: a survey on the final week of life. Palliat Med 13:233-241

8. Mercadante S, Valle A, Porzio G, Costanzo BV, Fusco F, Aielli F, Adile C, Fara B, Casuccio A (2011) How do cancer patients receiving palliative care at home die? a descriptive study. J Pain Symptom Manage 42:702-709

9. Cheng WW, Willey J, Palmer JL, Zhang T, Bruera E (2005) Interval between palliative care referral and death among patients treated at a comprehensive cancer center. J Palliat Med 8:1025-1032

10. Costantini M, Beccaro M, Merlo F (2005) The last three months of life of Italian cancer patients. Methods, sample characteristics and response rate of he Italian Survey of the Dying of cancer (ISDOC). Palliat Med 19:628-638

11. WHO. http://www.who.int/cancer/palliative/definition/en/. Accessed 25 Aug 2012

12. Temel J, Greer J, Muzikansky A et al (2010) Early palliative care for patients with metastatic non-small-cell lung cancer. N Eng J Med 363:733-742

13. Saito AM, Landrum MB, Neville BA, Ayanian JZ, Earle C (2011) The effect on survival of continuing chemotherapy to near death. BMC Palliat Care 10:14

14. Mercadante S, Ferrera P, Casuccio A (2010) The use of opioids in the last week of life in an acute palliative care unit. Am J Hosp Palliat Care 27:514-517

15. Mercadante S, Villari P, Casuccio A (2011) An Italian survey on the attitudes in treating breakthrough cancer pain in hospice. Support Care Cancer 19:979-983

16. Mercadante S, Porzio G, Valle A, Fusco F, Aielli F, Costanzo V (2011) Palliative sedation in patients with advanced cancer followed at home: a systematic review. J Pain Symptom Manage 41:754-760

17. Mercadante S, Intravaia G, Villari P, Ferrera P, David F, Casuccio A (2009) Controlled sedation for refractory symptoms in dying patients. J Pain Symptom Manage 37:771-779

18. Bruera E, Hui D (2011) Palliative care units: the best option for the most distressed. Arch Intern Med 171:1601 